\title{
Traveling wave solutions for a nonlocal phase-field system
}

\author{
PETER W. BATES ${ }^{\dagger}$ \\ Department of Mathematics, Brigham Young University, Provo, UT 84602, USA \\ AND \\ FENGXIN CHEN \\ Division of Mathematics and Statistics, University of Texas at San Antonio, \\ 6900 North Loop 1604 West, San Antonio, TX 78249, USA
}

[Received 18 October 2000 and in revised form 14 February 2002]

\begin{abstract}
We establish the existence of traveling wave solutions for a phase-field system in which the order parameter has long-range self-interaction, when the latent heat coefficient is small. We also perform computational simulations to determine the nature of the wave.
\end{abstract}

Keywords: nonlocal phase transition, traveling waves, continuation.

\section{Introduction}

The purpose of this note is to establish the existence of a traveling wave solution to a nonlocal phase-field system and also, through computational simulations, examine the qualitative features of the wave.

With order parameter $\phi$ and scaled temperature $\theta$, our system takes the form

$$
\begin{aligned}
\frac{\partial \phi}{\partial t} & =(J * \phi-\phi)+f(\phi)+\theta, \\
\frac{\partial(\theta+l \phi)}{\partial t} & =\Delta \theta,
\end{aligned}
$$

where $J$ has unit integral, is non-negative, $J * \phi(x, t)=\int_{\mathbb{R}^{n}} J(x-y) \phi(y, t) \mathrm{d} y, f$ is a bistable nonlinearity and $l$ is a latent heat parameter.

The more usual (local) phase-field system,

$$
\begin{aligned}
\frac{\partial \phi}{\partial t} & =D \Delta \phi+f(\phi)+\theta, \\
\frac{\partial(\theta+l \phi)}{\partial t} & =\Delta \theta,
\end{aligned}
$$

has recently received considerable attention (see [7-9, 13, 15, 20], for instance) with the basic existence and uniqueness theory given by C. Elliott and S. Zheng in [12]. The existence and uniqueness of the solution to the initial value problem for the system (1.1)-(1.2) are studied in [1].

\footnotetext{
†Email: peter@math.byu.edu

*Corresponding author: Email: feng@math.utsa.edu
} 
The local phase-field system (1.3)-(1.4) was postulated as a phenomenological model by Langer in [19] and further discussed by Fix in [14]. Some of the references cited above provide evidence that the system produces qualitatively realistic behavior in comparison with experiments using materials undergoing phase transformations.

Notice that (1.2) is simply the heat equation with an additional term, $l \frac{\partial \phi}{\partial t}$, which is a heat source/sink resulting from the latent heat of transition. The kinetics of equation (1.1) drive the order parameter to the two stable phases, one having a larger basin of attraction than the other depending on the temperature. The surface tension or 'neighbor effect' is modeled by the term $J * \phi-\phi$ in (1.1) or by $D \Delta \phi$ in the local phase-field system. With this self-interaction, the tendency is for the order parameter at each spatial location to evolve to the most stable phase, as dictated by the temperature. On the other hand, as mentioned above, the temperature changes through the latent heat of transition and so there is some inhibition to phase change near critical temperature.

These systems are successful in duplicating transition events such as nucleation or spinodal decomposition because they are more than merely phenomenological. They are in fact both gradient flows of appropriate free energy functionals. Thus, if these functionals do indeed represent the energy of a material sample, then the evolutions predicted by the respective phase-field systems are consistent with the second law of thermodynamics, and so the models are physically realistic at a more fundamental level. It was this observation that led to the important work of Fife and Penrose in [20] and [13]. We give a formal derivation of (1.1)-(1.2), following their derivation of a generalization of the local system, but starting with a free energy functional that incorporates long-range self-interaction of the order parameter. In fact the first term in the expression for $\tilde{E}(\phi, u)$ in (1.5) below, which is the source of the nonlocality, was proposed by van der Waals in his fundamental paper [21] but then, for simplicity, it was replaced by the approximation $D \int|\nabla \phi|^{2} / 2$, which has now become the standard interaction term. An expression for the free energy incorporating long-range interaction in generalized Ising systems is derived from a microscopic viewpoint in [2] and [3].

To derive (1.1)-(1.2), consider a material in which the order parameter, $\phi$, describing phase has long-range interaction. We assume that the material can exist in two stable phases, which we take to be represented by $\phi= \pm 1$ when the temperature is zero. With $u$ representing temperature relative to zero, we assume (consistent with [21] and [2]) that the total free energy takes the form

$$
\tilde{E}(\phi, u)=\frac{1}{4} \int_{\Omega} \int_{\Omega} j(x-y)(\phi(x)-\phi(y))^{2} \mathrm{~d} x \mathrm{~d} y+\int_{\Omega}\left(W(\phi)+u^{2} / 2\right) \mathrm{d} x .
$$

Here, $j$ is the translationally invariant interaction coefficient, which is taken to be nonnegative with positive integral, and $W$ is a double-well potential having minima at the two stable phases, $\phi= \pm 1$.

Note that if one assumes that the interaction coefficient $j$ is extremely short-ranged then one may be somewhat justified in replacing $j(x-y)(\phi(x)-\phi(y))^{2}$ by $j(x-y)|\nabla \phi(x) \cdot(x-y)|^{2}$. With $\Omega=\mathbb{R}^{n}$, putting $z=x-y$ and integrating with respect to $z$ converts the first term of $\tilde{E}$ to $D \int|\nabla \phi|^{2} / 2$, provided $j$ is isotropic. Here, $d=\frac{n}{2} \int j(z) z_{1}^{2} \mathrm{~d} z$. With this approximation one would start with a free energy of the form

$$
\frac{D}{2} \int_{\Omega}|\nabla \phi|^{2} \mathrm{~d} x+\int_{\Omega}\left(W(\phi)+u^{2} / 2\right) \mathrm{d} x
$$

In this note we choose not to make this simplifying approximation and proceed with an analysis based on $\tilde{E}$. 
Consistent with thermodynamics, we assume that $\phi$ and $u$ evolve according to a gradient flow for $\tilde{E}$. First we change variables in order to more clearly incorporate the requirement that the total internal energy be conserved.

The internal energy density $e$ is defined as

$$
e=u+\bar{l} \phi,
$$

where $\bar{l}$ is a latent heat coefficient. We write the free energy in terms of $\phi$ and $e$ :

$$
E(\phi, e)=\tilde{E}(\phi, e-\bar{l} \phi) .
$$

We require

$$
\frac{\partial(\phi, e)^{T}}{\partial t}=-\operatorname{grad} E(\phi, e) .
$$

To ensure that total internal energy is conserved, and to keep the resulting evolution equation as simple as possible and physically reasonable (e.g. the long-range interaction is negligible at large distances), we use the metric for $(\phi, e) \in L^{2} \times H_{0}^{-1}$, where $H_{0}^{-1}$ is the set of distributions in the dual of $H^{1}$ with mean value zero. Note that the inner product in $H_{0}^{-1}$ is given by

$$
\langle\xi, \eta\rangle_{H_{0}^{-1}} \equiv\langle X, \eta\rangle_{L^{2}} \quad \text { for } \eta \in L^{2},
$$

where $X$ has mean zero and $-\Delta X=\xi$

Formally, taking the gradient of $E$ in the usual way but using the metric of $H_{0}^{-1}$ for the second component, (1.7) becomes

$$
\begin{aligned}
\gamma_{1} \frac{\partial \phi}{\partial t} & =\int_{\mathbb{R}^{n}} j(x-y) \phi(y) \mathrm{d} y-\left(\int_{\mathbb{R}^{n}} j\right) \phi-W^{\prime}(\phi)+\bar{l}(e-\bar{l} \phi), \\
\gamma_{2} \frac{\partial e}{\partial t} & =\Delta(e-\bar{l} \phi),
\end{aligned}
$$

where $\gamma_{1}$ and $\gamma_{2}$ are relaxation parameters, included as weights in the metric. Dividing by $\bar{j} \equiv$ $\int_{\mathbb{R}^{n}} j(x) \mathrm{d} x$, rescaling time to absorb the factor $\bar{j} / \gamma_{1}$ in the first equation, and rescaling space by a factor of $\sqrt{\gamma_{2} \bar{j} / \gamma_{1}}$, results in the system (1.1)-(1.2), where $J(x)=\bar{j}^{(n-2) / 2} j(\sqrt{\bar{j}} x)$ has unit integral, $J * \phi(x, t)=\int_{\mathbb{R}^{n}} J(x-y) \phi(y, t) \mathrm{d} y, l=\bar{l}^{2} / \bar{j}, f(\phi)=W^{\prime}(\phi) / \bar{j}$, and $\theta=\bar{l} u / \bar{j}$. Note that through the divergence theorem, assuming proper behavior at infinity or insulated boundary in a finite domain, integrating (1.9) over the domain shows that total internal energy is constant in time. A similar analysis using the energy given by (1.6) results in the system (1.3)-(1.4).

Here our main concern is the existence of traveling wave solutions for the system (1.1)-(1.2) and so we take $n=1$. The reduction from the higher-dimensional problem follows by assuming the wave depends upon a moving scalar variable and by redefining $J$ in the convolution after integrating over directions orthogonal to the direction of the wave.

In the case at hand we no longer have the regularizing effect of a parabolic-like equation for the order parameter since $\phi \rightarrow J * \phi-\phi$ is a bounded operator. Moreover, we do not have a comparison principle for this system, as we would in the isothermal case, (1.1) with $\theta$ constant. In some ways one may not expect to see traveling waves because the dissipation mechanisms in the two equations 
are significantly different, one being through a bounded operator and one through the unbounded Laplace operator. Nevertheless, using the continuation method, we are able to prove the existence of traveling wave solutions to (1.1)-(1.2) when latent heat $l$ is small. This is still not entirely trivial since the Fredholm theory must be shown to apply in this situation.

The isothermal case, in which we have a single equation for $\phi$, is studied in [5] and [10], where existence, uniqueness, and asymptotic stability of monotone waves are established (see also [11] and [18]). Interesting related equations also arise if one continues the Taylor expansion of $(\phi(x)-\phi(y))$ in the energy to a finite order beyond the first or one truncates an expansion of the operator $\phi \rightarrow$ $(J * \phi-\phi)$ in Fourier space (see [8] and [4]). One obtains a differential operator of arbitrarily high order, and traveling waves for the resulting equation or system with sufficiently small coefficients are exhibited in [4] (see also [6]).

For the system (1.1)-(1.2) on $\mathbb{R}^{1}$ we assume

$\left(\mathbf{H}_{1}\right) \quad J \in C^{1}(\mathbb{R})$ is nonnegative with $\int_{\mathbb{R}} J \mathrm{~d} x=1$.

$\left(\mathbf{H}_{2}\right) f \in C^{2}(\mathbb{R})$ is bistable, with exactly three zeros \pm 1 , and an intermediate point $q$. Also, assume there exists an interval $\left[\alpha_{1}, \alpha_{2}\right]$ containing $q$ such that $f^{\prime}(\phi) \geqslant 0$ for $\phi \in\left[\alpha_{1}, \alpha_{2}\right]$ and $f^{\prime}(\phi)<0$ for $\phi \notin\left[\alpha_{1}, \alpha_{2}\right]$.

The function $f(\phi)=\rho(\phi-q)\left(1-\phi^{2}\right)$ with $-1<q<1$ is a typical example.

We seek solutions to (1.1)-(1.2) of the form $\phi=\phi(x-c t), \theta=\theta(x-c t)$ for some constant $c \in \mathbb{R}$, with $\phi$ and $\theta$ satisfying certain conditions at infinity. In the moving frame given by $z=x-c t$, the system becomes

$$
\begin{aligned}
c \phi^{\prime}+(J * \phi-\phi)+f(\phi)+\theta & =0, \\
c(\theta+l \phi)^{\prime}+\theta^{\prime \prime} & =0,
\end{aligned}
$$

and integrating the second equation gives (for $c \neq 0$ )

$$
(\theta+l \phi)+\frac{\theta^{\prime}}{c}=e_{\infty}
$$

where $e_{\infty}$ is a constant.

To select suitable conditions at infinity, we choose the temperature at one end of the wave, say $\theta(-\infty)=\theta^{-} \in\left(\Theta^{-}, \Theta^{+}\right)$, where $\Theta^{ \pm}$are the local minimum and maximum values of $-f$. Then $\phi$ at $z=-\infty$ is determined from (1.10) by $f\left(\phi^{-}\right)+\theta^{-}=0$, since the other terms in (1.10) vanish at $\pm \infty$. We choose $\phi^{-}$to be the left-most such value of $\phi$. Now equation (1.12) implies that, if $c \neq 0$, $(\phi, \theta)$ at $z=+\infty$, lies on the line

$$
\theta+l \phi=e_{\infty}\left(=\theta^{-}+l \phi^{-}\right) .
$$

Again using (1.10), we choose $\left(\phi_{l}^{+}, \theta_{l}^{+}\right)$as the point where this line intersects the right ascending branch of $-f$. The subscripts indicate that these values depend upon $l$ (see Fig. 1). If $l$ is too large, depending on $\theta^{-}$, then there will be no intersection and hence no choice for the boundary conditions at the right end of the wave.

Note that in the case $c=0,(1.11)$ implies that $\theta$ is constant and $\left(\phi_{l}^{+}, \theta_{l}^{+}\right)$is the point where the line $\theta=\theta^{-}$intersects the right ascending branch of $-f$. Since this point is independent of $l$, we call it $\left(\phi^{+}, \theta^{-}\right)$. In general, we impose the asymptotic conditions

$$
\begin{gathered}
\phi(-\infty)=\lim _{z \rightarrow-\infty} \phi(z)=\phi^{-}, \quad \phi(+\infty)=\lim _{z \rightarrow+\infty} \phi(z)=\phi_{l}^{+}, \\
\theta(-\infty)=\lim _{z \rightarrow-\infty} \theta(z)=\theta^{-}, \quad \theta(+\infty)=\lim _{z \rightarrow+\infty} \theta(z)=\theta_{l}^{+} .
\end{gathered}
$$




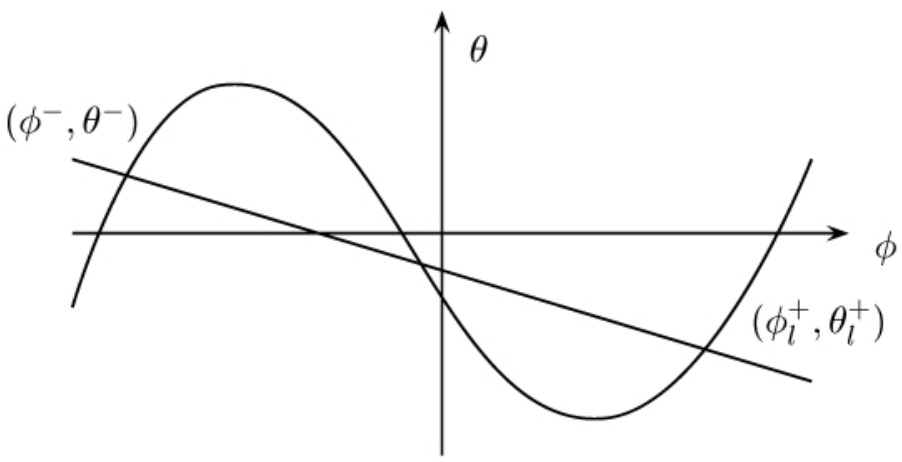

FIG. 1. Function $\theta=-f(\phi)$ and the line $\theta+l \phi=e_{\infty}$.

The points $\left(\phi^{-}, \theta^{-}\right)$and $\left(\phi_{l}^{+}, \theta_{l}^{+}\right)$are the two stable spatially homogeneous equilibria of the system (1.1)-(1.2) with the given value $e_{\infty}$ of the internal energy density. Note that a traveling wave solution is a triple $(\phi, \theta, c)$, giving the phase and temperature profiles and the wave speed $c$ satisfying (1.11) and (1.12). We say the traveling wave solution $(\phi, \theta, c)$ connects the two stable equilibria $\left(\phi^{-}, \theta^{-}\right)$and $\left(\phi_{l}^{+}, \theta_{l}^{+}\right)$if (1.14) and (1.15) hold.

We have the following theorem.

THEOREM 1.1 For any $\theta^{-} \in\left(\Theta^{-}, \Theta^{+}\right)$, there exists an $l_{0}>0$ such that, for all $0 \leqslant l<l_{0}$, system (1.1)-(1.2) admits a traveling wave solution $(\phi, \theta, c)$ connecting $\left(\phi^{-}, \theta^{-}\right)$and $\left(\phi_{l}^{+}, \theta_{l}^{+}\right)$.

REMARK 1.1 The traveling wave solutions to (1.1)-(1.2) are obviously not unique since any spatial shift of such a solution is also a traveling wave solution but it would be interesting to know if there were others. Given $e_{\infty}$, our proof provides local uniqueness of $(\phi, \theta, c)$ in $C^{1} \times C^{2} \times \mathbb{R}$ (up to translation) and we conjecture that the wave is globally unique for small values of $l$.

In Section 2 we prove Theorem 1.1 using the Implicit Function Theorem. In Section 3, we give some numerical simulations for the traveling wave solutions of (1.1)-(1.2). It is to be expected that monotonicity of the wave is lost due to latent heat release and the computational experiments bear this out.

\section{Proof of Theorem 1.1}

We first establish some lemmas useful in proving Theorem 1.1. Note that the case $c=0$, which occurs only if $\theta \equiv \frac{1}{\phi^{+}-\phi^{-}} \int_{\phi^{-}}^{\phi^{+}} f(s) \mathrm{d} s$, results in the scalar equation (1.1) and is treated in [5] and [10]. Therefore, we assume that $c \neq 0$ and without loss of generality, $c>0$. Solving (1.12) for $\theta(z)$ with $e_{\infty}$ given in (1.13), gives

$$
\theta(z)=\theta^{-}+l c \int_{-\infty}^{z} \mathrm{e}^{-c(z-\xi)}\left[\phi^{-}-\phi(\xi)\right] \mathrm{d} \xi .
$$

Combining with (1.10), we are led to consider

$$
c \phi^{\prime}+J * \phi-\phi+f(\phi)+\theta^{-}+l c \int_{-\infty}^{z} \mathrm{e}^{-c(z-\xi)}\left[\phi^{-}-\phi(\xi)\right] \mathrm{d} \xi=0 .
$$


When $l=0$, equation $(2.2)$ reduces to

$$
c \phi^{\prime}+J * \phi-\phi+f(\phi)+\theta^{-}=0 .
$$

This is the equation obtained from (1.1) for $\theta \equiv \theta^{-}$in a traveling wave frame. Equation (2.3) has been extensively studied in [5] and [10]. Note that with our restrictions on $\theta^{-}$, the function $f(\phi)+\theta^{-}$has the same properties as $f(\phi)$, apart from the locations of the three zeros. Because of this, there is no loss of generality if we assume that $\phi^{-}=-1$ and $\theta^{-}=0$, which we do from now on. By rescaling $\phi$ and redefining $f$, we may also assume that $\phi^{+}=1$.

For the existence and asymptotic behavior of solutions of (2.3) (taking $\theta^{-} \equiv 0$ ), we have the following properties.

LEMMA 2.1 $[5,10]$ There is a unique solution $\left(\phi_{0}, c_{0}\right)$ of $(2.3)$ satisfying $\phi_{0}(0)=0$ and $\lim _{z \rightarrow \infty} \phi_{0}( \pm z)= \pm 1$. Moreover, if $c_{0} \neq 0, \phi_{0}$ is of class $C^{2}, \phi_{0}^{\prime}(z)>0$ for all $z \in \mathbb{R}$, and

$$
c_{0}=-\frac{\int_{-1}^{1} f(\phi) \mathrm{d} \phi}{\int_{-\infty}^{\infty}\left(\phi_{0}^{\prime}(z)\right)^{2} \mathrm{~d} z} .
$$

We will use the Implicit Function Theorem to obtain a solution of (2.2) for small $l$. For that purpose, define

$$
X_{0}=\{\text { uniformly continuous functions on } \mathbb{R} \text { which vanish at } \pm \infty\},
$$

equipped with the supremum norm. Note that dual $X_{0}^{*}$, the space of bounded linear functionals on $X_{0}$, consists of Borel measures.

Suppose that $\left(\phi_{0}, c_{0}\right)$ is the solution of (2.3) given in Lemma 2.1. Let $\mathcal{L}=\mathcal{L}\left(\phi_{0}, c_{0}\right)$ be the linear operator defined in $X_{0}$ by

$$
D(\mathcal{L})=X_{1}:=\left\{v \in X_{0}: v^{\prime} \in X_{0}\right\}
$$

and

$$
\mathcal{L} \psi \equiv c_{0} \psi^{\prime}+J * \psi-\psi+f^{\prime}\left(\phi_{0}\right) \psi
$$

for $\psi \in D(\mathcal{L})$

We first prove that 0 is an isolated eigenvalue. Let $\zeta$ be a smooth function satisfying $\zeta(x)=0$ for $x \leqslant-1, \zeta(x)=1$ for $x \geqslant 1$ and $\zeta^{\prime}(x)>0$ for $x \in(-1,1)$ and let $s(x)=f^{\prime}(-1)(1-\zeta(x))+$ $f^{\prime}(1) \zeta(x)$. Define an operator $\mathcal{L}_{0}$ on $X_{0}$ with domain $D\left(\mathcal{L}_{0}\right)=X_{1}$ by $\mathcal{L}_{0} \psi=c_{0} \psi^{\prime}+J * \psi-\psi+$ $s(x) \psi$ for $\psi \in D\left(\mathcal{L}_{0}\right)$. Then we have the following lemma.

LEMMA $2.2\left\{\lambda: \operatorname{Re} \lambda>\max \left\{f^{\prime}(-1), f^{\prime}(1)\right\}\right\} \subset \rho\left(\mathcal{L}_{0}\right)$.

Proof. Let $\lambda \in \mathbb{C}$ with $\operatorname{Re} \lambda>\max \left\{f^{\prime}(-1), f^{\prime}(1)\right\}$. For any $g \in X_{0}$, consider the equation

$$
\mathcal{L} \psi-\lambda \psi=g(x)
$$

Let $\eta(x)=\psi\left(-c_{0} x\right)$. Then (2.6) becomes

$$
\left.\eta^{\prime}(x)+\left(1-s\left(-c_{0} x\right)+\lambda\right)\right) \eta(x)=\bar{J} * \eta(x)-g\left(-c_{0} x\right),
$$


where $\bar{J}(x)=\left|c_{0}\right| J\left(-c_{0} x\right)$. Or equivalently,

$$
\eta(x)=(\Lambda \eta)(x)-\int_{0}^{\infty} \mathrm{e}^{-\int_{x-\xi}^{x} h(\tau) \mathrm{d} \tau} g\left(-c_{0}(x-\xi)\right) \mathrm{d} \xi,
$$

where $\Lambda$ is an integral operator with the kernel

$$
\Lambda(x, y)=\int_{0}^{\infty} \mathrm{e}^{-\int_{x-\xi}^{x} h(\tau) \mathrm{d} \tau} \bar{J}(x-\xi-y) \mathrm{d} \xi,
$$

and $\left.h(x)=1-s\left(-c_{0} x\right)\right)+\lambda$, that is,

$$
(\Lambda \eta)(x)=\int_{-\infty}^{\infty} \Lambda(x, y) \eta(y) \mathrm{d} y
$$

for $\eta \in X_{0}$. We have $\|\Lambda\|_{X_{0}}<1$ since $\operatorname{Re} h(x)>1$. Therefore 1 is in $\rho(\Lambda)$. That is $\lambda \in \rho\left(\mathcal{L}_{0}\right)$.

Suppose $q \in X_{0}$ and define a bounded linear operator $B$ on $X_{0}$ by $B u=q u$ for $u \in X_{0}$. Then we have the following lemma.

Lemma 2.3 Let $\lambda \in \rho\left(\mathcal{L}_{0}\right)$. Then $B\left(\lambda-\mathcal{L}_{0}\right)^{-1}$ is a compact operator on $X_{0}$.

Proof. Let $E$ be a bounded closed set in $X_{0}$. Since $\left\|\left(\lambda-\mathcal{L}_{0}\right)^{-1} f\right\|_{X_{1}} \leqslant C\|f\|_{X_{0}}$ and $\left(\lambda-\mathcal{L}_{0}\right)^{-1} E$ is bounded in $X_{1}$ and therefore it is compact in $C([-n, n])$ for all $n>0$. A diagonal argument completes the proof.

Now take $q(x)=f^{\prime}(\phi(x))-s(x)$ and note that $\mathcal{L}=\mathcal{L}_{0}+B$. By Theorem A.1 (p. 136) in [16], we deduce that the half plane $\left\{\lambda: \operatorname{Re} \lambda>\max \left\{f^{\prime}(-1), f^{\prime}(1)\right\}\right\}$ consists entirely of normal points of $\mathcal{L}$, i.e. points in the resolvent set or eigenvalues of finite algebraic multiplicity.

LEMMA 2.4 If $c_{0} \neq 0$, then 0 is an eigenvalue of $\mathcal{L}$ and it is algebraically simple.

Proof. Since $\left(\phi_{0}, c_{0}\right)$ satisfies (2.3) and since $\lim _{z \rightarrow \infty} \phi_{0}( \pm z)= \pm 1, p=\phi_{0}^{\prime}$ vanishes at $\pm \infty$ is a positive eigenfunction of $\mathcal{L}$ in $X_{0}$ with corresponding eigenvalue 0 .

To show that 0 is a geometrically simple eigenvalue we use a maximum principle. Suppose that $\mathcal{L} v=0$ with $v \in X_{0}$ and assume, without loss of generality, that $v\left(x_{0}\right)>0$ for some $x_{0}$. For $t<0$ let $w_{t} \equiv \phi_{0}+t v$, so that $\min w_{t}(x)<0$ if $|t|$ is sufficiently large. Let $x_{t}$ be a point at which this minimum is achieved. We have $\mathcal{L} w_{t}=0$ and since $w_{t}$ is not constant, $\left(J * w_{t}-w_{t}\right)\left(x_{t}\right)>0$ for at least one choice of the point of minimum, $x_{t}$. Hence, $f^{\prime}\left(\phi_{0}\right) w_{t}\left(x_{t}\right)>0$. Because $f^{\prime}\left(\phi_{0}( \pm \infty)\right)>0$, we conclude that $x_{t} \in[-M, M]$ for some fixed $M$, independent of $t$.

Let $T \equiv \sup \left\{t<0: \min w_{t}(x)<0\right\}$. By passing to the limit as $t \rightarrow T^{-}$, we have $\mathcal{L} w_{T}=0$, $w_{T} \geqslant 0$ on $\mathbb{R}$ and $w_{T}\left(x_{T}\right)=0$ for some $x_{T} \in[-M, M]$. This implies $\left(J * w_{T}-w_{T}\right)\left(x_{T}\right)=0$, and hence that $w_{T} \in X_{0}$ is constant, namely zero. Therefore, $\phi_{0}$ and $v$ are linearly dependent.

To show algebraic simplicity, suppose that there is a $v$ such that $\mathcal{L} v=-\phi_{0}^{\prime}$ and recall that $\phi_{0}^{\prime}(x)>0$ on $\mathbb{R}$. Let $w_{\tau} \equiv v+\tau \phi_{0}^{\prime}$ and note that for $\tau>0$ and sufficiently large, $w_{\tau}>0$ on $[-M, M]$ and, as we argued above, $w_{\tau}>0$ on $\mathbb{R}$. Taking the infimum $T$ of all such $\tau$ produces a function $w_{T} \geqslant 0$ on $\mathbb{R}$ and a point $x_{T}$ where this function is zero. Then $0 \geqslant J * w_{T}\left(x_{T}\right)=$ $\mathcal{L} w_{T}\left(x_{T}\right)=-\phi_{0}^{\prime}\left(x_{T}\right)<0$ provides the contradiction which establishes the result. 
Proof of Theorem 1.1. Suppose $\left(\phi_{0}, c_{0}\right)$ is the solution given in Lemma 2.1.

(1) If $c_{0}=0$. Then $\left(\phi_{0}(x), \theta^{-}\right)$is a trivial traveling wave solution of (1.1)-(1.2) connecting the two stable equilibria for any value of $l$.

(2) Suppose $c_{0}>0$. Let $\zeta(x)$ be a smooth function such that $\zeta(x)=0$ for $x \leqslant 0, \zeta(x)=1$ for $x \geqslant 2$, and $0 \leqslant \zeta^{\prime}(x) \leqslant 1$ for all $x$. Define $\chi_{l}(x)=\phi_{l}^{+} \zeta(x)-(1-\zeta(x))$ for $l \geqslant 0$. Recall that $\phi_{0}(-\infty)=\phi^{-}=-1$ and $\phi_{0}(\infty)=\phi_{0}^{+}$, so that

$$
\left(\phi_{0}+\chi_{l}-\chi_{0}\right)(-\infty)=\phi^{-} \text {and }\left(\phi_{0}+\chi_{l}-\chi_{0}\right)(\infty)=\phi_{l}^{+} .
$$

Consider the operator $G:\left(X_{1} \times\left(-c_{0}, \infty\right)\right) \times \mathbb{R} \rightarrow X_{0} \times \mathbb{R}$ defined by

$$
\begin{aligned}
G(w, l)= & \left(\left(c_{0}+c\right)\left(\psi+\phi_{0}+\chi_{l}-\chi_{0}\right)^{\prime}+J *\left(\psi+\phi_{0}+\chi_{l}-\chi_{0}\right)\right. \\
& -\left(\psi+\phi_{0}+\chi_{l}-\chi_{0}\right)+f\left(\psi+\phi_{0}+\chi_{l}-\chi_{0}\right) \\
& \left.+l\left(c_{0}+c\right) \int_{-\infty}^{x} \mathrm{e}^{-\left(c_{0}+c\right)(x-\xi)}\left[-1-\left(\psi+\phi_{0}+\chi_{l}-\chi_{0}\right)\right] \mathrm{d} \xi, \psi(0)\right)
\end{aligned}
$$

for $w=(\psi, c) \in X_{1} \times\left(-c_{0}, \infty\right)$. Then $G$ is of class $C^{1}, G(0,0)=(0,0)$ and

$$
\frac{\partial G}{\partial w}(0,0)=\left[\begin{array}{cc}
\mathcal{L} & \phi_{0}^{\prime} \\
\delta & 0
\end{array}\right],
$$

where $\delta$ is the $\delta$-function. We show that $\frac{\partial G}{\partial w}(0,0)$ is invertible from $X_{1} \times \mathbb{R}$ to $X_{0} \times \mathbb{R}$. Consider the equation

$$
\frac{\partial G}{\partial w}(0,0)\left[\begin{array}{l}
\psi \\
c
\end{array}\right]=\left[\begin{array}{l}
h \\
b
\end{array}\right], \quad \text { for }\left[\begin{array}{l}
h \\
b
\end{array}\right] \in X_{0} \times \mathbb{R}
$$

i.e.

$$
\begin{aligned}
\mathcal{L} \psi+c \phi_{0}^{\prime} & =h, \\
\psi(0) & =b .
\end{aligned}
$$

By Theorem 5.28, p. 239 of [17], $\mathcal{L}$ has closed range, and so by the Fredholm Alternative, (2.11) is solvable if and only if $h-c \phi_{0}^{\prime}$ annihilates the null space of $\mathcal{L}^{*}$, the adjoint of $\mathcal{L}$. Since this null space is one-dimensional and is generated by a linear functional, $\phi^{*}$, this amounts to

$$
c\left\langle\phi^{*}, \phi_{0}^{\prime}\right\rangle=\left\langle\phi^{*}, h\right\rangle,
$$

where $\langle\cdot, \cdot\rangle$ is the duality pairing. Since $\left\langle\phi^{*}, \phi_{0}^{\prime}\right\rangle \neq 0$, by the simple algebraic multiplicity of $\phi_{0}^{\prime}$, the value of $c$ is uniquely determined by (2.13). Now the solution to (2.11) is determined up to an additive multiple of $\phi_{0}^{\prime}$. This multiple is determined uniquely by (2.12) since $\phi_{0}^{\prime}(0)>0$. Therefore $\frac{\partial G}{\partial w}(0,0)$ is invertible.

By the Implicit Function Theorem, there is an $l_{0}>0$ such that for all $0 \leqslant l<l_{0}$ there is a unique solution $\left(\psi_{l}, c(l)\right)$ of $G((\psi, c), l)=(0,0)$. Let

$$
c_{l}=c_{0}+c(l), \quad \phi_{l}(x)=\psi+\phi_{0}+\chi_{l}-\chi_{0},
$$

and

$$
\theta_{l}(x)=l c_{l} \int_{-\infty}^{x} \mathrm{e}^{-c_{l}(x-\xi)}\left[-1-\phi_{l}(\xi)\right] \mathrm{d} \xi
$$


Then $\left(\phi_{l}\left(x-c_{l} t\right), \theta_{l}\left(x-c_{l} t\right)\right)$ is a traveling wave solution of (2.2) corresponding to the boundary condition (1.14)-(1.15) with $\left(\phi^{-}, \theta^{-}\right)=(-1,0)$, and satisfying $\phi(0)=0$.

(3) If $c_{0}<0$, a similar argument is used but the integrals are from $x$ to $\infty$ instead.

This completes the proof.

\section{Numerical results}

We conclude this paper with the results of some numerical experiments for system (1.1)-(1.2) with certain initial data $(\phi(x, 0), \theta(x, 0))$ and for various values of the latent heat coefficient $l$.

We choose $J(x)=\frac{1}{\sqrt{\pi}} \mathrm{e}^{-x^{2}}$ and $f(\phi)=(\phi-q)\left(1-\phi^{2}\right)$, with $q \in(-1,1)$. The initial data $\phi(x, 0)$ and $\theta(x, 0)$ are chosen to be piecewisely linear, $\phi(x, 0)$ connecting $\phi^{-} \equiv-1$ and $\phi_{l}^{+}$, and $\theta(x, 0)$ connecting $\theta^{-} \equiv 0$ and $\theta_{l}^{+}$. Recall that $\phi_{l}^{+}$and $\theta_{l}^{+}$are defined just before (1.14)-(1.15). We take

$$
\phi(x, 0)= \begin{cases}-1 & \text { if } x \leqslant-55 \\ -1+\frac{\phi_{l}^{+}+1}{10}(x+55) & \text { if }-55<x<-45 \\ \phi_{l}^{+} & \text {if } x \geqslant-45\end{cases}
$$

and

$$
\theta(x, 0)= \begin{cases}0 & \text { if } x \leqslant-55, \\ \frac{\theta_{l}^{+}}{10}(x+55) & \text { if }-55<x<-45, \\ \theta_{l}^{+} & \text {if } x \geqslant-45 .\end{cases}
$$

For computations with the usual phase-field system, (1.3)-(1.4), one can specify boundary conditions by linearizing the equation at the equilibria and using the resulting relationship between the solution to the linear equation and its derivative. In our case, we can not specify boundary conditions in that way. Instead, in order to observe traveling waves for (1.1)-(1.2), we choose a large spatial interval to begin with and assume that the boundary condition will not strongly affect the solution in a short spatial range. Therefore we choose the space interval to be $[-500,500]$ and impose Dirichlet-type boundary conditions:

$$
\begin{array}{r}
\phi(x)=-1, \quad \theta(x)=0, \text { for } x \leqslant-500 ; \\
\phi(x)=\phi_{l}^{+}, \quad \theta(x)=\theta_{l}^{+}, \text {for } x \geqslant 500 .
\end{array}
$$

We use an explicit scheme to solve the first equation and use an implicit scheme to solve the second equation. We used different grids as a check on the accuracy and the output presented here appears to be robust.

We observed traveling waves for various choices of $q$ and the latent heat coefficient $l$. Some results are shown in Figs 2-5.

It is interesting to see from Figs $2-5$ that as the latent heat coefficient $l$ increases the monotonicity of profiles is lost. This is physically sound due to the latent heat effect.

The numerical experiment suggests that the traveling waves are stable. Theoretically, the uniqueness (modulo a spatial shift) and stability still remain open. Also we do not know the asymptotics or long time behavior of solutions of initial value problems when $l$ is fairly large. 


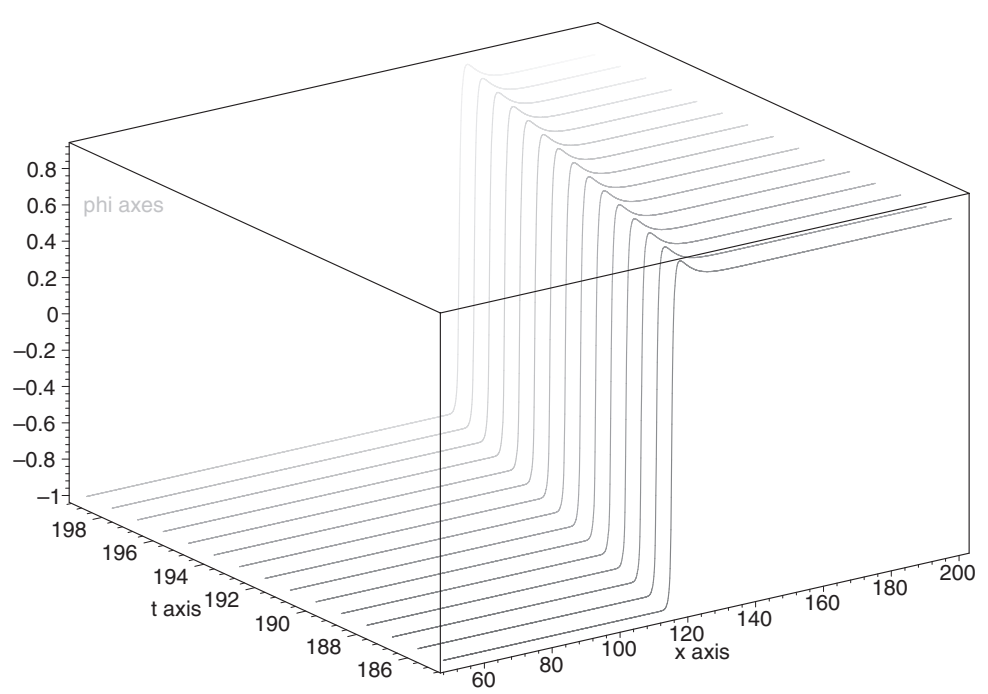

FIG. 2. Solution $\phi$ when $f(\phi)=\left(\phi^{2}-1\right)(0.5-\phi)$ and latent heat coefficient $l=0.06$.

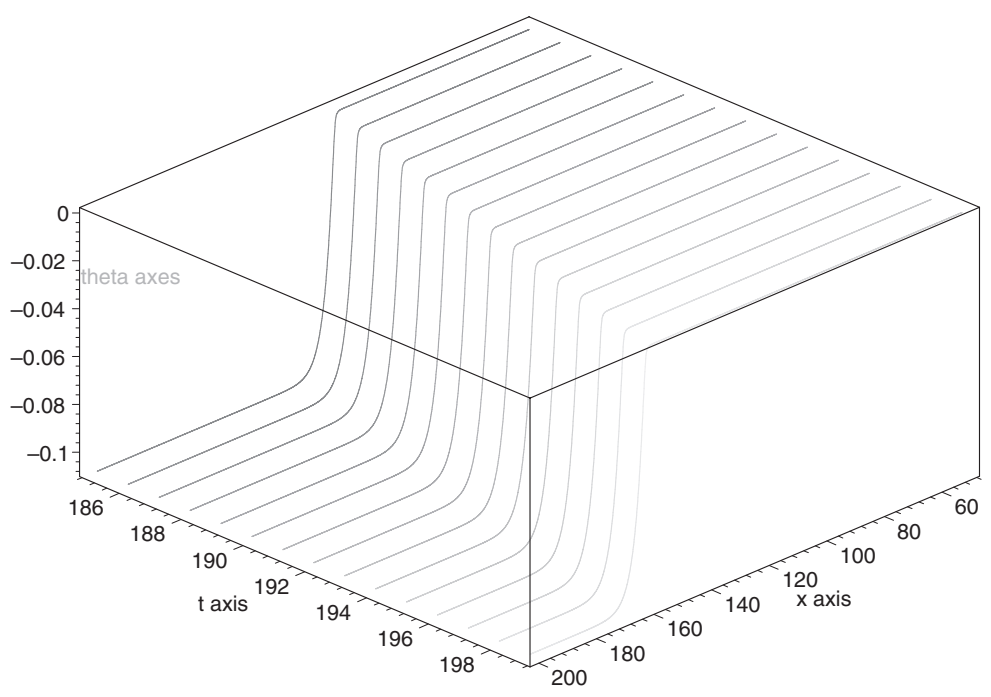

FIG. 3. Solution $\theta$ when $f(\phi)=\left(\phi^{2}-1\right)(0.5-\phi)$ and latent heat coefficient $l=0.06$.

\section{Conclusion}

A phase-field system is postulated which incorporates long-range interaction for the order parameter with bistable kinetics. The evolution for the order parameter is coupled to the usual diffusive heat equation which includes a heat source/sink corresponding to latent heat effects. Traveling waves are shown to exist for sufficiently small values of the latent heat coefficient. We conjecture that they exist up to a certain critical value of the latent heat coefficient, depending on the temperature specified at one end of the wave. 


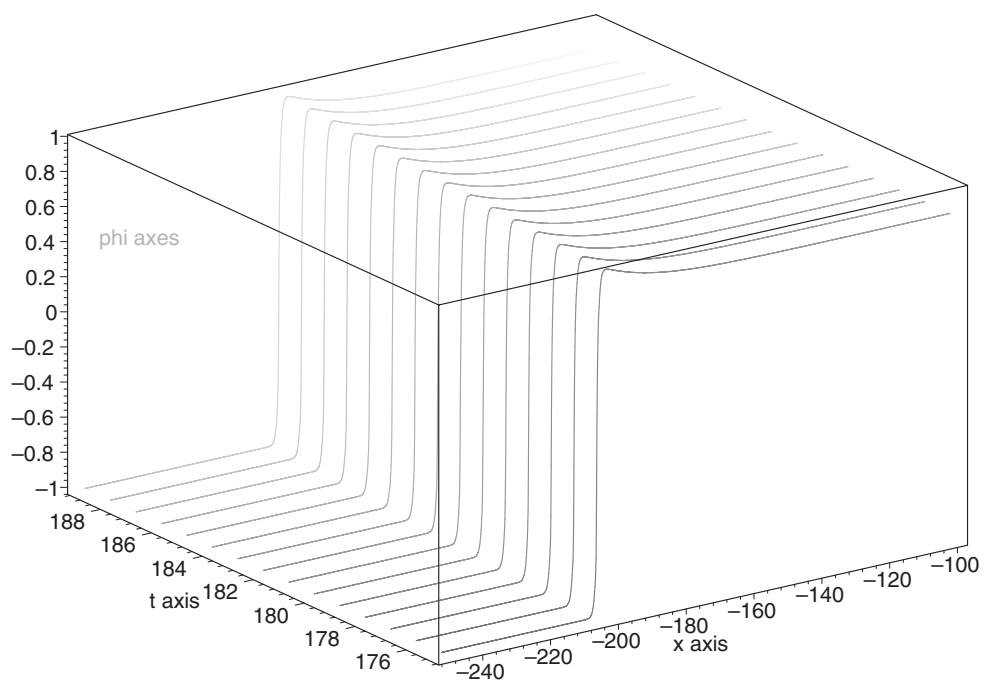

FIG. 4. Solution $\phi$ when $f(\phi)=\left(\phi^{2}-1\right)(0.2-\phi)$ and latent heat coefficient $l=0.1$.

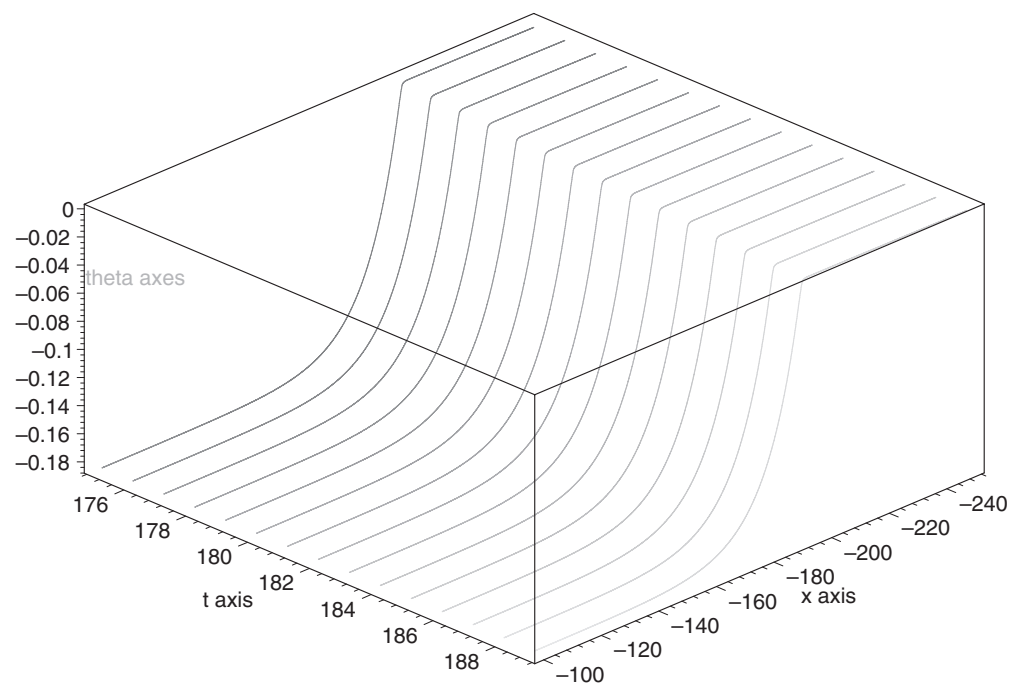

FIG. 5. Solution $\theta$ when $f(\phi)=\left(\phi^{2}-1\right)(0.2-\phi)$ and latent heat coefficient $l=0.1$.

Although local uniqueness (up to translation) of the wave with a given temperature at one end is shown, it is not known if it is globally unique. Nor is stability established, though our computational experiments suggest that this is the case.

Computations further suggest, as is to be expected in a realistic model, that for larger values of the latent heat coefficient, the wave is not monotone near the interface due to the release of latent heat during solidification. 


\section{Acknowledgements}

PB has been partially supported in part by NSF awards DMS-9974340 and DMS 9970894.

\section{REFERENCES}

1. Bates, P. W., Chen, F., \& WANG, J. Global existence and uniqueness of solutions to a nonlocal phasefield system. In: BATES, P. W., ChOW, S.-N., LU, K. \& PAN, X. (eds), US-Chinese Conference on Differential Equations and Applications, International Press, Cambridge, MA (1997) pp. 14-21.

2. BATES, P. W. \& ChMAJ, A. An integrodifferential model for phase transitions: stationary solutions in higher space dimensions. J. Stat. Phys. 95, (1999) 1119-1139.

3. Bates, P. W. \& Chmaj, A. A discrete convolution model for phase transitions. Arch. Rational Mech. Anal. 150, (1999) 281-305.

4. Bates, P. W., Fife, P. C., Gardner, R. A., \& Jones, C. K. R. T. The existence of traveling wave solutions of a generalized phase-field model. SIAM J. Math. Anal. 28, (1997) 60-93.

5. Bates, P. W., Fife, P. C., Ren, X., \& WAnG, X. Traveling waves in a nonlocal model of phase transitions. Arch. Rat. Mech. Anal. 138, (1997) 105-136.

6. BATES, P. W. \& REN, X. Heteroclinic orbits for a higher order phase transition problem. Euro. J. Applied Math. 8, (1997) 149-163.

7. Caginalp, G. An analysis of a phase field model of a free boundary. Arch. Rat Mech. Anal. 92, (1986) 205-245.

8. Caginalp, G. \& Fife, P. C. Dynamics of layered interfaces arising from phase boundaries. SIAM J. Math. Anal. 48, (1988) 506-518.

9. Caginalp, G. \& Nishiura, Y. The existence of traveling waves for phase field equations and convergence to sharp interface models in the singular limit. Quar. Appl. Math. 49, (1991) 147-162.

10. CHEN, X. Existence, uniqueness and asymptotic stability of traveling waves in nonlocal evolution equations. Adv. Diff. Eqs. 2, (1997) 125-160.

11. de Masi, A., Orlandi, E., Presutti, E., \& Triolo, L. Uniqueness of the instanton profile and global stability in nonlocal evolution equations. Rend. Math. 14, (1994) 693-723.

12. Elliott, C. \& Zheng, S. Global existence and stability of solutions to the phase field equations. In: Hoffmann, K. H. \& Sprekels, J. (eds), Free Boundary Problems, International Series of Numerical Mathematics 95. pp. 46-58. Birkhauser, Basel (1990).

13. Fife, P. C. \& Penrose, O. Interfacial dynamics for thermodynamically consistent phase-field models with nonconserved order parameter. Electron. J. Diff. Eqns. (1995) 1-49.

14. FIX, G. J. Phase field methods for free boundary problems. In: FASANO, B. \& Primicerio, M. (eds), Free Boundary Problems: Theory and Applications. pp. 580-589. Pitman, London (1983).

15. FRIED, E. \& GURTIN, M. E. A phase-field theory for solidification based on a general anisotropic sharpinterface theory with interfacial energy and entropy. Physica D 91, (1996) 143-181.

16. Henry, D. Geometric Theory of Semilinear Parabolic Equations, Lect. Notes Math. 840. Springer, New York (1981).

17. Kato, T. Perturbation Theory for Linear Operators. Springer, New York (1982).

18. Katsoulakis, M. \& Souganidis, P. S. Interacting particle systems and generalized mean curvature evolution. Arch. Rat Mech. Anal. 127, (1994) 133-157.

19. Langer, J. S. Theory of the condensation point. Ann. Phys. 41, (1967) 108-157.

20. Penrose, O. \& Fife, P. C. Thermodynamically consistent models of phase-field type for the kinetics of phase transitions, interacting particle systems and generalized mean curvature evolution. Physica D 43, (1990) 44-62.

21. VAN DER WAALS, J. D. The thermodynamic theory of capillarity flow under the hypothesis of a continuous variation in density. Verhandelingen der Koninklijke Nederlandsche Akademie van Wetenschappen te Amsterdam 1, (1893) 1-56. 